\title{
Retraction of: Astrobiology, 2016;16(5):335-347; DOI: 10.1089/ast.2015.1394
}

\begin{abstract}
O N 27 APRIL 2016, after acceptance and publication of the above article, the senior author learned that the University of Florida (UF) Institutional Review Board (IRB) review and approval process for using human subjects in a minimal risk scientific research project was not properly followed. Since that date, the senior author has worked closely with the UF/IRB team, the editorial staff at the journal Astrobiology, and the publisher Mary Ann Liebert, Inc. to discuss and rectify the situation. At the time the research was performed (Nov. 2012 through March 2013), the three human volunteers mentioned in the article were clearly informed of the intended use of the samples outlined in the study, were informed that the results (if meritorious) would be published in a peer-reviewed scientific journal, and were informed that all samples would be destroyed by autoclaving and then incineration at the conclusion of the research. No identifiable metadata related to the volunteers was recorded. The authors stand by the original results and interpretations of the research, but must now retract the paper because the guidelines for working with human subjects were not followed. We apologize for the error, and look forward to reworking the manuscript into a new paper with the human data removed, and submitting it to an appropriate peerreviewed astrobiology publication venue.
\end{abstract}

Astrobiology commends Dr. Schuerger for his honesty and transparency. 